\title{
Aflatoxin influences achalasia symptomatology
}

\author{
SHENGLI LIN*, PINGTING GAO*, QUANLIN LI*, YIQUN ZHANG, JIANWEI HU, MINGYAN CAI, \\ WENZHENG QIN, LILI MA, ZHONG REN, ZHEN ZHANG, XIANLI CAI, \\ LIQING YAO, WEIFENG CHEN and PINGHONG ZHOU
}

Endoscopy Center and Endoscopy Research Institute, Zhongshan Hospital, Fudan University, Shanghai 200032, P.R. China

Received May 5, 2019; Accepted December 6, 2019

DOI: $10.3892 / \mathrm{mmr} .2020 .10914$

\begin{abstract}
Achalasia is characterized by impaired swallowing due to lower esophageal sphincter (LES) dysfunction and an increased risk of esophageal carcinoma. Aflatoxin is a known carcinogen. Esophageal retention is relieved by per oral endoscopic myotomy (POEM), which lowers the esophageal cancer risk. The present study determined whether aflatoxin is involved in the pathogenesis of achalasia or esophageal cancer. A total of 75 patients with achalasia were prospectively enrolled from a tertiary center. Aflatoxin levels in their esophageal contents were measured using ELISA, and esophageal mucosal specimens were immunohistochemically evaluated for Ki67 and p53 expression prior to and 3 months after POEM. The effect of aflatoxin on esophageal contractility was assessed using murine specimens. Aflatoxin was detected in 67 patients before POEM and only 2 patients after POEM. The number of Ki67- and p53-immunopositive cells in the esophageal mucosa significantly decreased after POEM: [Ki67: $27.8 \%$ (95\% confidence interval (CI), 25.98-29.70) vs. $20.7 \%$ (95\% CI, 19.78-24.03), $\mathrm{P}=0.04$ and p53: $2.14 \%$ (95\% CI, $1.85-2.41)$ vs. $1.45 \%$ (95\% CI, $1.22-1.68), \mathrm{P}=0.03]$. In vitro experiments revealed that $500 \mathrm{ng} / \mathrm{ml}$ aflatoxin significantly increased the amplitude $(\mathrm{P}<0.05)$ and frequency $(\mathrm{P}<0.05)$ of spontaneous LES contractions compared with the control group. These increases were blocked by co-treatment with atropine sulfate $(\mathrm{P}<0.05)$, but not with a nitric oxide synthase inhibitor $(\mathrm{P}>0.05)$. Aflatoxin was found in most patients with achalasia and was eliminated following POEM. Reduced Ki67 and p53 expression after POEM indicated a decreased risk of carcinogenesis. Aflatoxin accumulation increased LES
\end{abstract}

Correspondence to: Professor Pinghong Zhou or Professor Weifeng Chen, Endoscopy Center and Endoscopy Research Institute, Zhongshan Hospital, Fudan University, 180 Fenglin Road, Shanghai 200032, P.R. China

E-mail: zhou.pinghong@zs-hospital.sh.cn

E-mail: chen.weifeng@zs-hospital.sh.cn

\section{${ }^{*}$ Contributed equally}

Key words: per oral endoscopic myotomy, achalasia, aflatoxin, lower esophageal sphincter contractility via cholinergic signaling. Therefore, aflatoxin may maintain achalasia symptoms and increase esophageal cancer risk.

\section{Introduction}

Achalasia, with an annual incidence of 1/100,000 in Western countries, is a rare motility disease characterized by impaired swallowing due to dysfunction of the lower esophageal sphincter (LES) $(1,2)$. The condition is associated with an increased risk of esophageal carcinoma $(3,4)$. Histopathological analyses of myotomy specimens from patients with idiopathic achalasia demonstrate a degradation of ganglion cells in the esophageal myenteric plexus $(5,6)$ and inflammatory cell infiltration. The etiology of achalasia is unknown, but has been postulated to be related to autoimmunity, neurodegeneration and viral immunity (7).

The predominant symptoms of achalasia are dysphagia and regurgitation. In addition, persistent esophageal distension due to food and fluid retention can lead to fungal and bacterial overgrowth, and impaired clearance of gastric contents (8). Acid regurgitation can cause chronic inflammation, dysplasia and even cancer. Meijssen et al (9) reported that the risk of esophageal cancer is 33 times greater in patients with achalasia compared with the general population. Streitz et al (10) reported that the incidence of squamous cell carcinoma was $88 / 100,000$ in the patients with achalasia in their study, which represents a 14.5 times greater risk than that in the general population after adjustments for age and sex. In a recent study, Tustumi et al (11) performed a systematic review and meta-analysis that showed that achalasia cardia is associated with an increased risk of esophageal cancer, highlighting the need for strict endoscopic surveillance in patients with achalasia.

A potential contributor to the development of esophageal cancer in patients with achalasia is aflatoxin (AF) (12). AF is one of the most potent toxic, carcinogenic, teratogenic and immunosuppressive substances that is present naturally in certain foods, particularly improperly stored foods, such as corn, rice, peanuts, wheat and a variety of spices (13). AFs comprise a group of closely related mycotoxins that are produced as secondary metabolites by several fungi, namely Aspergillus (A.) flavus, A. nomius and A. parasiticus (14-16). Although the major AF subtypes $\left(B_{1}, B_{2}, G_{1}\right.$ and $\left.G_{2}\right)$ are often found together in varying proportions in different foods, $A F B_{1}$ 
is the predominant subtype with the most potent carcinogenic effect (17).

Since 2010, per oral endoscopic myotomy (POEM) has been used as an effective treatment option to relieve esophageal food retention in patients with achalasia (18), and is increasingly replacing pneumatic dilatation, Botox injection and Heller myotomy as a treatment for achalasia (19-22). Furthermore, Minami et al (23) reported that POEM may reduce the risk of esophageal carcinoma in patients with achalasia.

On this basis, it was hypothesized that an agent present in the food retained in the esophagus may be responsible for the subsequent development of carcinomas in patients with achalasia (24). The present study was designed to determine whether AFs are present in the esophageal contents of patients with achalasia and whether AFs are related to the symptomatology of achalasia, particularly the cancer risk.

\section{Materials and methods}

Study design and patient population. The present single-center prospective study consecutively enrolled 75 patients (age range, 14-81 years; 34 males and 41 females) who underwent POEM at the Endoscopy Center and Endoscopy Research Institute, Zhongshan Hospital, Fudan University, between January 2016 and June 2016. Patients were eligible for enrollment in the study if they were 14-90 years of age and had recurrent/persistent symptoms of achalasia with an Eckardt symptom score of $\geq 4$. The Eckardt score is the sum of the symptom scores for dysphagia, regurgitation, chest pain and weight loss (25). The diagnosis of achalasia was based on the absence of peristalsis and the presence of impaired LES relaxation on established tests (barium swallow, manometry or esophagogastroduodenoscopy). The exclusion criteria were pseudo-achalasia, megaesophagus (diameter $>7 \mathrm{~cm}$ ), and severe cardiopulmonary disease or other serious disease posing unacceptable surgical risk (26). Patients with mild or moderate esophagitis that did not induce esophageal stricture were not excluded. In addition, 30 healthy volunteers (age, 23-45 years; 18 males and 12 females) were recruited following healthy examination at the Endoscopy Center and Endoscopy Research Institute, Zhongshan Hospital, Fudan University, between April 2016 and September 2016. Esophageal contents samples obtained from healthy volunteers were used as controls. The baseline characteristics of the patients are given in Table I.

All patients and volunteers provided written informed consent prior to their inclusion into the study. Written informed consent was obtained from the parents/guardians of all minors included in the present study. The study was approved by the ethics committee of Zhongshan Hospital, Fudan University (approval no. B2012-089).

Calculation of LES pressure. The pre- and post-myotomy LES pressures were recorded using a high-resolution manometry system (Sierra Scientific Instruments Inc.), as described previously (27). In brief, the high-resolution manometry assembly was placed transnasally, and the manometric catheter was positioned to record from the hypopharynx to the stomach with $\sim 5$ intragastric sensors. Manometric assessments were performed with the patient in a supine position and after $\geq 6 \mathrm{~h}$ of fasting. The manometric protocol included a $5 \mathrm{~min}$ period to measure the basal gastroesophageal junction pressure; 10 water swallows of $5 \mathrm{ml}$ each; and 1 water swallow each of 1 (dry), 10 and $20 \mathrm{ml}$. All manometric analyses were performed using Mano-View (version 3.0; Medtronic); the data tracings were evaluated in the color pressure topography mode and referenced to the intragastric pressure.

Treatment and sample collection. All patients were treated using POEM, which was performed by experienced endoscopists at the Endoscopy Center and Endoscopy Research Institute, Zhongshan Hospital, Fudan University. The patients underwent gastroscopy examination again at 3 months after POEM. A proton pump inhibitor (usually omeprazole) and antibiotics (usually second-generation cephalosporins) were administered for 1-2 days during the fasting period after POEM. Patients were usually discharged 2-3 days after POEM if they were able to swallow liquids and had no other complaints, such as fever.

Prior to the POEM procedure, samples of the esophageal contents were collected from the patients with achalasia by performing saline irrigation of the esophageal lumen, followed by fluid suction into a sterile container. Using the same method, esophageal samples were obtained from the healthy volunteers in the control group. During the POEM procedure, two biopsy specimens of the esophageal mucosa were collected from each patient. The biopsy sites were located $2 \mathrm{~cm}$ from the cardia, within the esophagus.

Measurement of AF concentration in esophageal contents. The esophageal luminal contents were examined for AFs by using ELISA (cat. no. E030502; Shanghai Bangyi Biological Technology Co. Ltd.). This kit can detect AFs $B_{1}, B_{2}, G_{1}$ and $G_{2}$. The assays were conducted according to the manufacturer's instructions. Residual food in the esophagus was aspirated using a regular gastroscope during the endoscopic examination before POEM. If the residual food was primarily solid, $\sim 250 \mathrm{ml}$ of sterile normal saline was used to irrigate the esophagus until it was clean. The original volume of the samples used in for ELISA was usually 20-30 ml. Samples were prepared as follows: $35 \mathrm{ml}$ methanol was added to the $15 \mathrm{ml}$ original sample and shaken at $20-25^{\circ} \mathrm{C}$ for $15 \mathrm{~min}$ with a shaker; the sample was centrifuged at $1500 \mathrm{x} g$ at $20-25^{\circ} \mathrm{C}$ for $5 \mathrm{~min}$; $1 \mathrm{ml}$ supernatant was mixed with $1 \mathrm{ml}$ deionized water; $50 \mu \mathrm{l}$ diluted supernatant was added to each well and incubated for $2 \mathrm{~h}$ at $20-25^{\circ} \mathrm{C}$ prior to analysis by ELISA.

Immunohistochemical analysis of esophageal biopsy specimens. A total of two $4 \mathrm{~mm}$-long esophageal mucosal specimens were collected during each POEM procedure. The specimens were fixed using $4 \%$ formalin at $20-25^{\circ} \mathrm{C}$ for $8-12 \mathrm{~h}$, paraffin-embedded, cut into $3 \mu \mathrm{m}$ thick sections, mounted on silane-coated glass slides, dried, deparaffinized with xylene and rehydrated using a graded ethanol series. For antigen retrieval, the specimens were boiled for $20 \mathrm{~min}$ in $10 \mathrm{mM}$ monosodium citrate buffer ( $\mathrm{pH}$ 6.0) for Ki67 immunostaining or $10 \mathrm{mM}$ Tris/EDTA buffer (pH 9.0) for p53 immunostaining. To quench endogenous peroxidase, the slides were incubated in a $0.5 \%$ solution of $\mathrm{H}_{2} \mathrm{O}_{2}$ in phosphate-buffered citric acid for $20 \mathrm{~min}$ at $20^{\circ} \mathrm{C}$, washed 3-5 times with TBS ( $\mathrm{pH} 7.4$ ), and incubated again with TBS containing $10 \%$ non-immune rabbit serum 
Table I. Patient characteristics before and after POEM.

\begin{tabular}{|c|c|}
\hline Characteristic & Value \\
\hline Age, years; median [IQR] (range) & $40.00[26.0-51.0](14.0-81.0)$ \\
\hline$<18, \mathrm{n}(\%)$ & $2.00(2.7)$ \\
\hline$\geq 18$ and $<60, \mathrm{n}(\%)$ & $65.00(86.7)$ \\
\hline$\geq 60, \mathrm{n}(\%)$ & $8.00(10.7)$ \\
\hline \multicolumn{2}{|l|}{ Sex, n $(\%)$} \\
\hline Male & $34.00(45.3)$ \\
\hline Female & $41.00(54.7)$ \\
\hline Disease duration, years; median [IQR] (range) & $8.00[4.0-10.0](0.5-30.0)$ \\
\hline Sigmoid-type esophagus, n (\%) & $9.00(12)$ \\
\hline Pretreatment, n (\%) & $21.00(28)$ \\
\hline Balloon dilatation, $\mathrm{n}(\%)$ & $9.00(12)$ \\
\hline Stent placement, n (\%) & $4.00(5.3)$ \\
\hline Botox treatment, $\mathrm{n}(\%)$ & $3.00(4)$ \\
\hline Heller myotomy, n (\%) & $4.00(5.3)$ \\
\hline POEM, n (\%) & $4.00(5.3)$ \\
\hline Balloon dilatation and POEM, n (\%) & $1.00(1.3)$ \\
\hline Balloon dilatation and Heller myotomy, n (\%) & $1.00(1.3)$ \\
\hline Stent and Botox treatment, n (\%) & $1.00(1.3)$ \\
\hline Duration of POEM, min; median [IQR] (range) & $52.00[32.0-69.0](16.0-154.0)$ \\
\hline Pre-POEM LES pressure, mmHg; median [IQR] (range) & $25.10[17.3-35.8](3.9-49.5)$ \\
\hline Pre-POEM Eckardt score; median [IQR] (range) & $7.00[6.0-8.0](4.0-10.0)$ \\
\hline Pre-POEM aflatoxin, ng/ml; median [IQR] (range) & $13.14[9.3-15.7](0.0-36.8)$ \\
\hline Negative, n (\%) & $8.00(10.7)$ \\
\hline Positive, n (\%) & $67.00(89.3)$ \\
\hline Post-POEM LES pressure, $\mathrm{mmHg}$; median [IQR] (range) & $10.1[8.0-13.2](5.0-16.2)$ \\
\hline Post-POEM Eckardt score; median [IQR] (range) & $1.00[1.0-2.0](0.0-7.0)$ \\
\hline Post-POEM aflatoxin, ng/ml; median [IQR] (range) & $0.15[0.0-0.0](0.0-6.3)$ \\
\hline Negative, n (\%) & $73.00(97.3)$ \\
\hline Positive, n (\%) & $2.00(2.7)$ \\
\hline
\end{tabular}

IQR, interquartile range; POEM, per oral endoscopic myotomy; LES, lower esophageal sphincter.

(Dako; Agilent Technologies, Inc.) with 10\% human plasma (Ki67; Dako; Aglient Technologies, Inc.) or 5\% bovine serum albumin (p53; Dako; Aglient Technologies, Inc.) at $20-25^{\circ} \mathrm{C}$ for $30 \mathrm{~min}$. Afterwards, the slides were incubated with the primary antibodies anti-Ki67 (cat. no. M724029; MIB-1; Dako; Agilent Technologies, Inc.) and anti-p53 (cat. no. NCL-L-p53-DO7; DO-7; Leica Biosystems $\mathrm{GmbH}$ ) at 1:100 dilution for $12 \mathrm{~h}$ at $0-4^{\circ} \mathrm{C}$. Next, the slides were incubated with biotin-labeled rabbit anti-mouse secondary antibody (cat. no. DS9800; Leica Microsystems $\mathrm{GmbH}$ ) at $20-25^{\circ} \mathrm{C}$ for $8 \mathrm{~min}$ and a streptavidin-horseradish peroxidase complex (cat. no. DS9800; Leica Biosystems GmbH). 3-Amino-9-ethylcarbazole was used as the substrate for detection for $5 \mathrm{~min}$ at $20-25^{\circ} \mathrm{C}$. The immunochemical staining was observed using a DM 2000 light microscope (magnification, x200; Leica Microsystems GmbH) and was analyzed by two experienced pathologists who were blinded to the clinical data. At least 300 nuclei were counted per section, and cells with moderate-to-intense nuclear staining were counted manually as immune-positive.
Animal experiments. A total of 12 male Wistar albino rats (weight, 280 g; age, 8 weeks; Beijing Vital River Laboratory Animal Technology Co., Ltd.) were used in the present study. The recommendations of the Guide for the Care and Use of Laboratory Animals of Zhongshan Hospital, Fudan University were followed, and the animal experiments were approved by the Medical Faculty Animal Care Committee of Zhongshan Hospital, Fudan University. The rats were housed at $18-25^{\circ} \mathrm{C}$ with $40-70 \%$ humidity and $12 \mathrm{~h}$ light/dark cycles, and fed water and food ad libitum. All the rats in the study were anesthetized prior to cervical dislocation. Ketamine $(100 \mathrm{mg} / \mathrm{kg})$ and xylazine $(10 \mathrm{mg} / \mathrm{kg})$ were administered by intraperitoneal injection to anesthetize the rats.

Drugs. AF $\mathrm{B}_{1}$, a nitric oxide (NO) synthase inhibitor [NG-nitro-L-arginine (L-NNA)], and atropine sulfate were obtained from Sigma-Aldrich (Merck KGaA). A 2 mg/ml stock solution of AF was prepared in dimethyl sulfoxide (which does not directly affect murine-isolated smooth muscle 
preparations) and stored at $0-4^{\circ} \mathrm{C}$. Other drugs were dissolved in ethanol and deionized water (1:1) as $1 \mathrm{mg} / \mathrm{ml}$ stock solutions, and were subsequently diluted in deionized water before use.

Isolated smooth muscle experiments. All 12 rats were sacrificed by means of cervical dislocation, and a $2 \mathrm{~cm}$-long esophageal specimen was excised from the gastro-esophageal junction through a $\sim 7 \mathrm{~cm}-$ long mid-abdominal incision. Fat and connective tissues were removed from the excised specimen, and the esophageal lumen was cleaned with Tyrode solution $\left(\mathrm{NaCl}, 8 \mathrm{~g} / \mathrm{l} ; \mathrm{CaCl}_{2}, 2.5 \mathrm{~g} / \mathrm{l} ; \mathrm{KCl}, 0.2 \mathrm{~g} / \mathrm{l} ; \mathrm{MgSO}_{4}\right.$, $0.1 \mathrm{~g} / 1 ; \mathrm{NaH}_{2} \mathrm{PO}_{4}, 0.05 \mathrm{~g} / \mathrm{l} ; \mathrm{NaHCO}_{3}, 1.0 \mathrm{~g} / \mathrm{l}$; and glucose, $1.0 \mathrm{~g} / \mathrm{l})$ previously aerated with $95 \% \mathrm{O}_{2}$ and $5 \% \mathrm{CO}_{2}$. Then, $1 \mathrm{~cm}$-long full-thickness esophageal segments were hung in a circular orientation in tissue baths filled with $10 \mathrm{ml}$ Tyrode solution at $37.0^{\circ} \mathrm{C}$. The upper ends of the esophageal segments were attached to an isometric transducer (cat. no. JZJ01; Chengdu Instrument Factory) and the lower ends preloaded with $1.5 \mathrm{~g}$ tension. The tissues were equilibrated for $20 \mathrm{~min}$. Tissue contraction was considered the reference response after equilibrium and at the beginning of each experiment. The tissues were equilibrated for $\sim 20$ min again before the addition of 5, 50 and $500 \mathrm{ng} / \mathrm{ml} \mathrm{AF}$. Isometric tension was recorded and analyzed using the RM6280 system (Chengdu Instrument Factory). To ensure maximal effect, the esophageal segments were incubated with each concentration of AF for $3 \mathrm{~min}$. Subsequently, $20 \mathrm{~min}$ before the final and highest dose of AF $(500 \mathrm{ng} / \mathrm{ml})$, the cholinergic inhibitor atropine sulfate $(0.2 \mu \mathrm{g} / \mathrm{ml})$ or the NO synthase inhibitor L-NNA $(0.2 \mu \mathrm{g} / \mathrm{ml})$ was added to the bath.

Statistical analysis. Data were expressed as mean \pm standard error of the mean. All statistical analyses were conducted using SPSS v13.0 software (SPSS, Inc.). Continuous data were compared using Student's t-test between two groups. One-way ANOVA followed by Bonferroni's test was applied to assess the differences among groups. The pre-operative values of AF, Ki67 and p53 were subtracted from the post-operative values to represent the changes of these indexes after POEM. Subsequently, bivariate Pearson correlation analysis was used to measure relationships between two variables. All tests were two sided, and $\mathrm{P}<0.05$ was considered to indicate a statistically significant difference.

\section{Results}

AF is found in esophageal contents and eliminated after POEM in most patients with achalasia. A total of 75 patients were enrolled in this study. Their baseline characteristics are shown in Table I. Before POEM, AF was detected in the esophageal contents of 67 of the 75 patients with achalasia. These samples exhibited a yellow-green fluorescence upon irradiation with $365 \mathrm{~nm}$ violet light, which indicated the presence of AF (Fig. 1A and B); in contrast, the control group did not show any fluorescence (Fig. 1C and D). Notably, at 3 months after POEM, AF was detected in only 2 samples, and both of these patients still had dysphagia and retention symptoms.

While performing the POEM procedure, retained foods were first removed from the esophagus (Fig. 2A). In one patient,
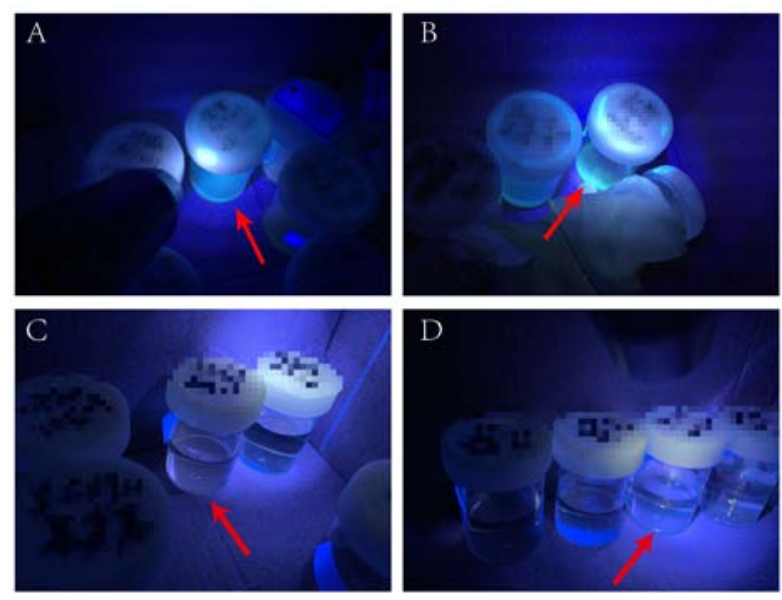

Figure 1. Fluorescence under 365-nm violet light. (A and B) Aflatoxincontaining samples of retained food exhibited yellow-green fluorescence upon irradiation with $365 \mathrm{~nm}$ violet light, while (C and D) control-group samples did not exhibit any fluorescence. Red arrows indicate which sample is being referred to in each panel.

after the esophagus had been cleared, a large sheet erosion was incidentally found in the esophageal mucosa, and this area was biopsied (Fig. 2B). The pathological report showed esophageal squamous epithelium with high-grade dysplasia, and the patient underwent endoscopic submucosal dissection in the Endoscopy Center and Endoscopy Research Institute, Zhongshan Hospital, Fudan University after a few weeks.

Reduced Ki67 and p53 immunoreactivity after POEM. To assess markers associated with cancer development, immunohistochemical analyses of the esophageal specimens was performed. The mean percentage of Ki67-immunopositive cells was significantly lower in the esophageal mucosa samples taken after POEM compared with the samples collected before POEM, with 27.8\% [95\% (CI), 25.98-29.70] before compared with $20.7 \%$ (95\% CI, 19.78-24.03) after ( $\mathrm{P}=0.04$; Fig. 3). Similarly, the mean percentage of p53-positive cells significantly decreased after POEM [2.14\% (95\% CI, 1.85-2.41) vs. $1.45 \%$ (95\% CI, 1.22-1.68); P=0.03; Fig. 4].

However, no correlation was observed between AF and Ki67 ( $R=-0.142, P=0.223$; Fig. $S 1 A)$, or between AF and p53 ( $R=0.179, P=0.124$; Fig. S1B) prior to POEM. Similarly, following POEM, no correlation was observed between AF and $\mathrm{Ki67}(\mathrm{R}=-0.174, \mathrm{P}=0.135$; Fig. $\mathrm{S} 2 \mathrm{~A})$, or between $\mathrm{AF}$ and p53 ( $\mathrm{R}=0.098, \mathrm{P}=0.401$; Fig. $\mathrm{S} 2 \mathrm{~B})$.

AF increases the amplitude and frequency of LES contractions in vitro. To determine whether AF affected LES contractions in patients with achalasia, in vitro experiments were performed using esophageal segments excised from adult Wistar albino rats. It was found that both the frequency and amplitude of LES contractions significantly increased after the application of $500 \mathrm{ng} / \mathrm{ml} \mathrm{AF} \mathrm{B}$ (Fig. 5A and B; $\mathrm{P}<0.05$ ). This AF-induced increase in esophageal tissue contractions was blocked by cholinergic inhibition. The bath application of atropine sulfate $(0.2 \mu \mathrm{g} / \mathrm{ml})$ significantly inhibited the amplitude (Fig. 6A) and frequency (Fig. 6B) of LES contractions in the presence of AF (both $\mathrm{P}<0.05$ ). However, the same dose of the NO synthase inhibitor L-NNA had no such effect. Altogether, these data 

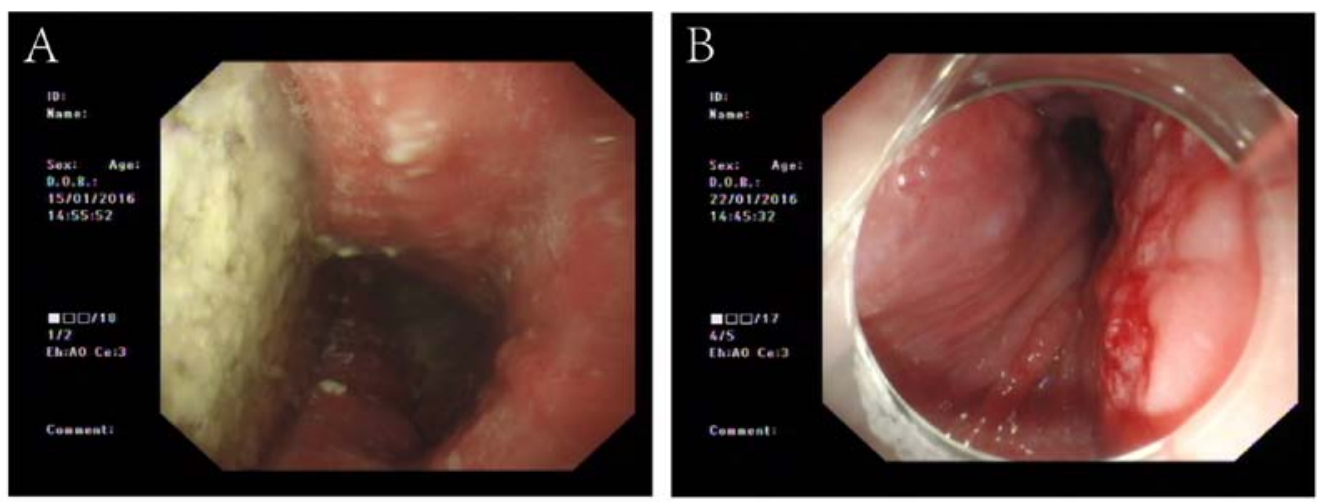

Figure 2. Gastroscopy images of achalasia. (A) Gastroscopy showing food retention and inflammatory edema of the esophageal mucosa in a patient with achalasia at the beginning of the per oral endoscopic myotomy procedure. (B) Gastroscopy showing early esophageal carcinoma in a patient with achalasia; this patient underwent endoscopic submucosal dissection a few weeks later.

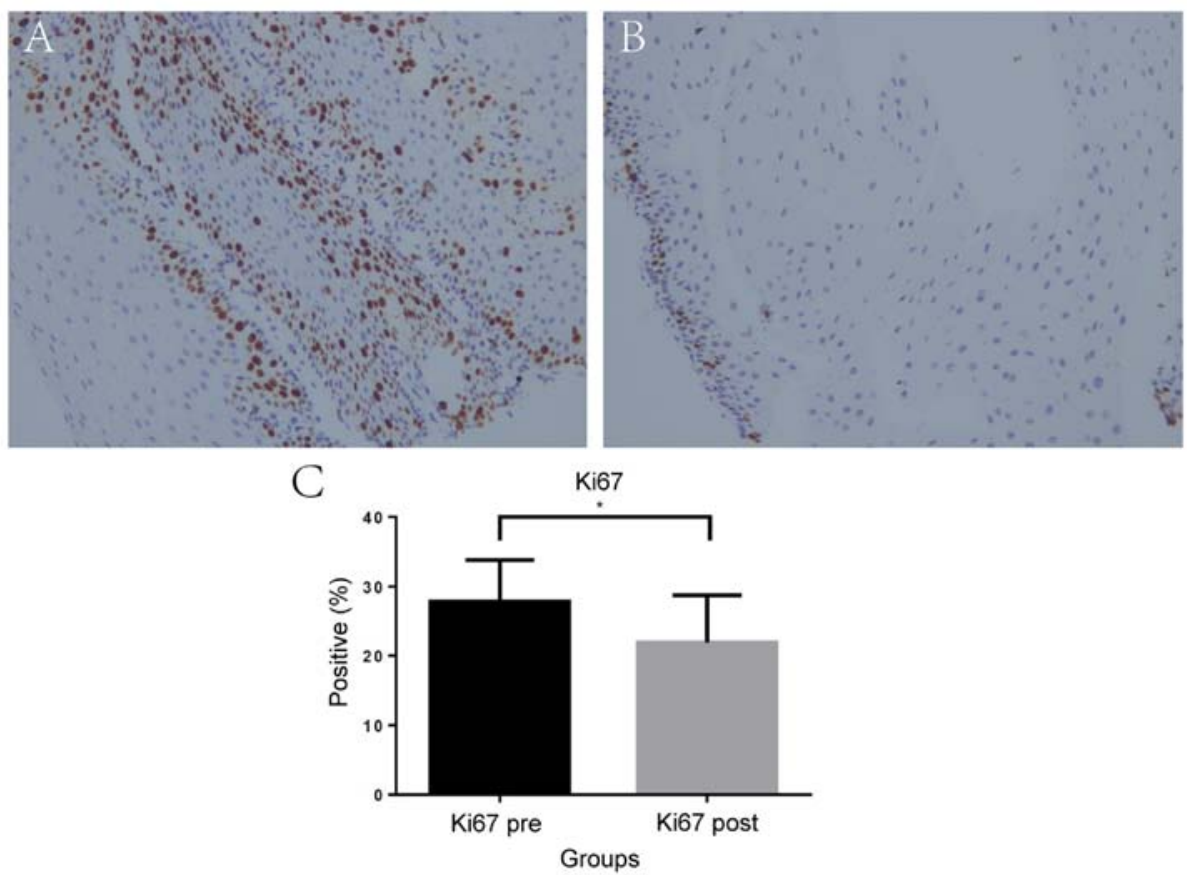

Figure 3. Immunohistochemical staining for Ki67 in the esophageal mucosa. Representative micrographs of Ki67 immunohistochemical analysis of specimens taken from patients with achalasia (A) before and (B) after POEM (magnification x200). (C) Quantification revealed a significant reduction in the percentage of Ki67-positive cells after POEM. " $\mathrm{P}<0.05$, as indicated. POEM, per oral endoscopic myotomy.

demonstrated that LES contractions increased in the presence of $\mathrm{AF}$, and that this increase was rapidly alleviated by the inhibition of cholinergic signaling.

\section{Discussion}

The present study measured the AF levels in the esophageal contents of patients with achalasia before and 3 months after they underwent POEM. The carcinogen AF was present in most patients with achalasia before POEM, and was absent in nearly all patients after POEM. In addition, the absence of AF paralleled the reductions in Ki67 and p53 immunoreactivity in the esophageal mucosa seen in biopsies, suggesting that $\mathrm{AF}$ accumulation in food contents retained within the esophagus may contribute to cancer development in patients with achalasia. In vitro studies revealed that AF accumulation was associated with an increase in the contractility of murine esophageal sections, and this increase was blocked by a cholinergic inhibitor. These findings suggested that AF is not only linked to markers of esophageal cancer in patients with achalasia but also a possible contributor to the symptomatology of achalasia via cholinergic signaling.

In the general population, AF is not detected in the blood using ELISA or high-performance liquid chromatography, and very low levels of AF $(<0.03 \mathrm{ng} / \mathrm{ml})$ are detected in urine samples (28). Environmental AF contamination reportedly ranges between $7.2-125.4 \mu \mathrm{g} / \mathrm{kg}$, which is normally too low to cause disease (29). While AF is not found in the esophagus in the general population, it was detected in most patients with achalasia enrolled in the present study. Importantly, minimal 

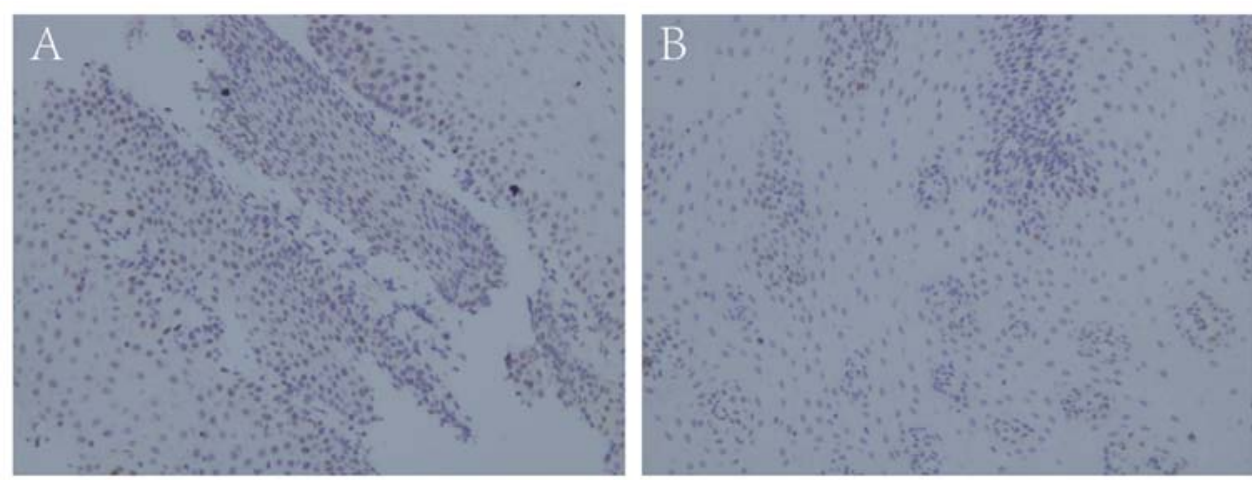

C p53

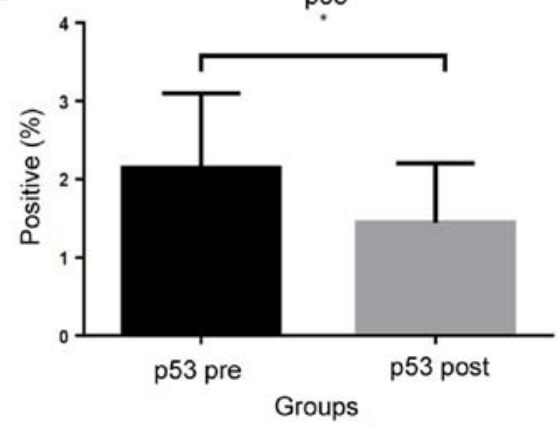

Figure 4. Immunohistochemical staining for p53 in the esophageal mucosa. Representative micrographs of p53 immunohistochemical analysis of specimens taken from patients with achalasia (A) before and (B) after POEM (magnification x200). (C) Quantification revealed a significant reduction in the percentage of p53-positive cells after POEM. " $\mathrm{P}<0.05$, as indicated. POEM, per oral endoscopic myotomy.

A

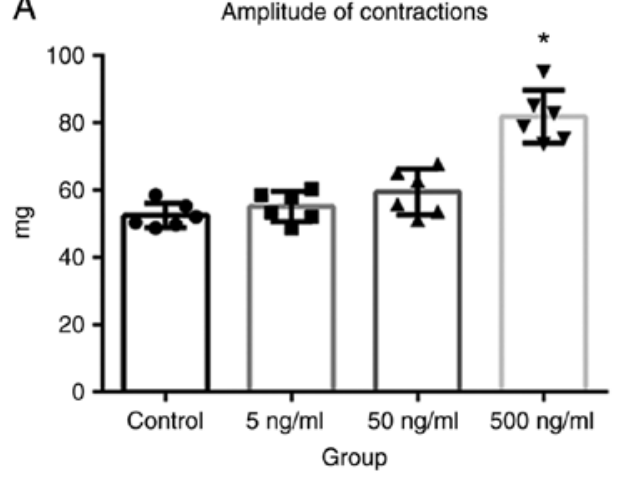

B

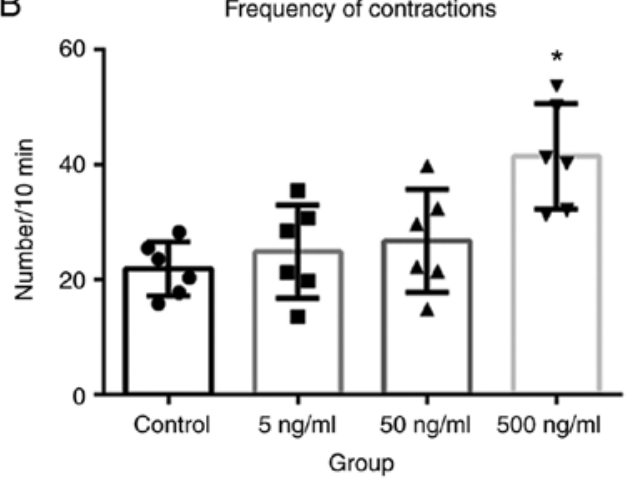

Figure 5. Amplitude and frequency under different levels of aflatoxin. Aflatoxin treatment increases the (A) amplitude and (B) frequency of murine lower esophageal sphincter contractions. Data are expressed as mean \pm standard error of the mean. ${ }^{*} \mathrm{P}<0.05$ vs. control.

levels of AF were detected in these patients after POEM, when the food retention had been alleviated. This is the first report, to the best of the authors' knowledge, documenting an association between AF and achalasia.

Fujii et al (30) reported higher Ki67 positivity in cancerous tissues compared with non-cancer tissues in patients with achalasia with concomitant esophageal squamous cancer. Leeuwenburgh et al (31) found that p53 overexpression might be an early marker of neoplastic progression in patients with achalasia. With this in mind, the present study performed immunohistochemical analyses of the esophageal mucosal specimens collected from the patients with achalasia. Notably, significant decreases in both these markers were observed after POEM. These findings implied that patients might be at a reduced risk of developing esophageal carcinoma after they have been treated with POEM and their symptoms have resolved. In the present study, AF was detected in the esophageal contents of 67 of the 75 patients with achalasia prior to POEM, but in only 2 patients following POEM. Therefore, it is not appropriate to directly use bivariate Pearson correlation analysis for the post-operative data. Thus, the pre-operative values of AF (also Ki67 and p53) were subtracted from the post-operative values to represent the changes of these indexes after POEM, then bivariate Pearson correlation analysis was used to assess the relationships between these indexes. However, no correlation was observed between AF and Ki67/p53 before or after POEM. The samples for AF ELISA were collected using gastroscopy. Sterile normal saline was used to flush food residue in the esophagus prior to its complete removal by suction. This may have contributed to 
A

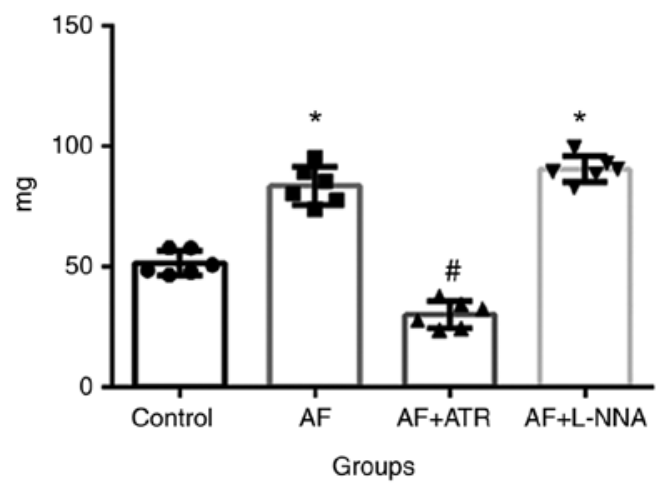

B

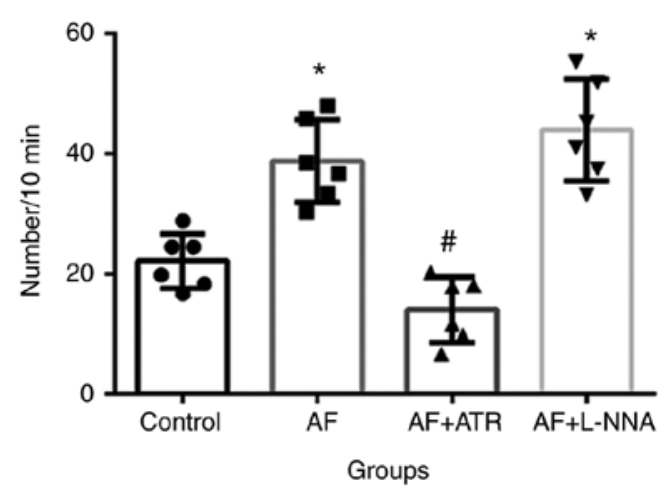

Figure 6. Amplitude and frequency under different types of solutions. (A) Amplitude and (B) frequency of murine LES contractions after the bath application of AF alone and with ATR or the nitric oxide synthase inhibitor l-NNA. The bath application of ATR (0.2 $\mu$ g/ml) significantly inhibited the (A) amplitude and (B) frequency of LES contractions in the presence of AF. However, the same dose of 1-NNA had no such effect. Data were expressed as mean \pm standard error of the mean. "P<0.05 vs. control; "P<0.05 vs. AF. LES, lower esophageal sphincter; AF, aflatoxin; ATR, atropine sulfate; 1-NNA, NG-nitro-1-arginine.

the negative result from dilution effects that may have lowered the AF concentration.

AF is known to induce both acute and chronic toxicity in numerous species (32). The in vitro experiments of the present study further demonstrated that AF directly influenced murine esophageal contractility, which is consistent with previous reports concerning the gastrointestinal systems of animals (33). AF has been shown to induce abdominal pain, diarrhea, vomiting and severe erosions (34). The present study found that a $500 \mathrm{ng} / \mathrm{ml}$ concentration of $\mathrm{AF} \mathrm{B}_{1}$ increased both the amplitude and frequency of LES contractions, and that these effects were driven by cholinergic signaling, as they were rapidly blocked by atropine sulfate.

Although the factors contributing to achalasia have not yet been fully elucidated, evidence suggests that ganglionitis due to aberrant immune responses to viral infections underlies the loss of esophageal neurons, particularly in genetically susceptible populations $(35,36)$. The loss of inhibitory tone and the subsequent retention of ingested food can lead to regurgitation, chest pain, dysphagia and weight loss, as well as an increased risk of esophageal carcinoma (25,37-39). Thus, the main pathophysiology of achalasia may be the degradation of ganglion cells in the esophageal myenteric plexus. Although the absence of NO synthase in the myenteric plexus of the LES has been proposed as the primary factor (40), the results of the present study suggested a more important role for the cholinergic system. However, other studies have reported that AF can inhibit NO synthase (41-43). Therefore, it is hypothesized that the AF-mediated inhibition of NO synthase may have a long-term effect on the LES, while AF has a direct and acute effect on LES via the cholinergic signaling pathway. However, verification using in vivo studies will make these results more convincing.

The present study has certain limitations. First, in the preliminary studies, a number of contaminants were found in food samples, including nitrates, Candida albicans, sterigmatocystin and Staphylococcus aureus. However, only AF was assessed in the present study, as AF is one of the most potent toxic and carcinogenic substances that is naturally present in improperly stored foods. Other carcinogens should be assessed in future studies. Second, the diet of the patients was not controlled, and the patients were not asked to provide diet information. Third, as mentioned earlier, saline irrigation and suction may not be an ideal method for the collection of esophageal contents due to dilution effects. An appropriate and efficient alternative method to collect samples for AF measurement without any bias needs to be found. Finally, animal models of achalasia are difficult to establish. Commonly used methods include esophageal ligation (44), but it is difficult to simulate the complex changes of achalasia using this method. Infection with Trypanosoma americanum (T. americanum) has been used to imitate the pathophysiology of achalasia in animals $(45,46)$, but this method might not be ethically acceptable, and T. americanum cannot be imported to China.

In conclusion, the findings of the present study demonstrated that AF accumulated in the esophageal contents of patients with achalasia and was eliminated after POEM. The reduced $\mathrm{Ki} 67$ and p53 expression after treatment in these patients showed that POEM may decrease the risk of esophageal neoplastic progression. It was hypothesized that the removal of AF after POEM may be the reason for the reduced expression of these biomarkers. Furthermore, the present study demonstrated that AF increased the contractility of the LES, and that this increase can be blocked by cholinergic inhibitors. Further studies are needed to elucidate how AF participates in the process of achalasia, the AF dose required to produce these effects, and whether esophageal AF accumulation is a cause or result of achalasia, or simply maintains the symptoms of achalasia. It is also hoped to establish a suitable animal model of achalasia to study the long-term effects of AF on the LES.

\section{Acknowledgements}

Not applicable.

\section{Funding}

The present study was supported by grants from The National Natural Science Foundation of China (grant nos. 81302098, $81370588,81470811,81401930$ and 81570595$)$ and The Natural Science Foundation of Shanghai (grant no. 13ZR1452300). 


\section{Availability of data and materials}

The datasets used and/or analyzed during the current study are available from the corresponding author on reasonable request.

\section{Authors' contributions}

SL, PG and QL collected the data, performed the experiments and wrote the manuscript. YZ, JH, MC, WQ, LM, ZR, ZZ, LY and $\mathrm{XC}$ collected and analyzed data. $\mathrm{PZ}$ and $\mathrm{WC}$ designed the study, recruited patients and volunteers and made critical revisions to the manuscript. All authors approved the final version of the manuscript.

\section{Ethics approval and consent to participate}

All patients and volunteers provided written informed consent prior to their inclusion into the study. Written informed consent was obtained from the parents/guardians of all minors included in the present study. The study was approved by the ethics committee of Zhongshan Hospital, Fudan University (approval no. B2012-089). The recommendations of the Guide for the Care and Use of Laboratory Animals of Zhongshan Hospital, Fudan University were followed, and the animal experiments were approved by the Medical Faculty Animal Care Committee of Zhongshan Hospital, Fudan University.

\section{Patient consent for publication}

Not applicable.

\section{Competing interests}

The authors declare that they have no competing interests.

\section{References}

1. Sadowski DC, Ackah F, Jiang B and Svenson LW: Achalasia: Incidence, prevalence and survival. A population-based study. Neurogastroenterol Motil 22: e256-e261, 2010.

2. Birgisson S and Richter JE: Achalasia in Iceland, 1952-2002: An epidemiologic study. Dig Dis Sci 52: 1855-1860, 2007.

3. Gennaro N, Portale G, Gallo C, Rocchietto S, Caruso V, Costantini M, Salvador R, Ruol A and Zaninotto G: Esophageal achalasia in the veneto region: Epidemiology and treatment Epidemiology and treatment of achalasia. J Gastrointest Surg 15: 423-428, 2011.

4. Marlais M, Fishman JR, Fell JM, Haddad MJ and Rawat DJ: UK incidence of achalasia: An 11 year national epidemiological study. Arch Dis Child 96: 192-194, 2011.

5. Clark SB, Rice TW, Tubbs RR, Richter JE and Goldblum JR: The nature of the myenteric infiltrate in achalasia: An immunohistochemical analysis. Am J Surg Pathol 24: 1153-1158, 2000.

6. Villanacci V, Annese V, Cuttitta A, Fisogni S, Scaramuzzi G, De Santo E, Corazzi N and Bassotti G: An immunohistochemical study of the myenteric plexus in idiopathic achalasia. J Clin Gastroenterol 44: 407-410, 2010.

7. Park W and Vaezi MF: Etiology and pathogenesis of achalasia: The current understanding. Am J Gastroenterol 100: 1404-1414, 2005.

8. Mohamed AA, Lu XL and Mounmin FA: Diagnosis and treatment of esophageal candidiasis: Current updates. Can J Gastroenterol Hepatol 20: 3585136, 2019.

9. Meijssen MA, Tilanus HW, van Blankenstein M, Hop WC and Ong GL: Achalasia complicated by oesophageal squamous cell carcinoma: A prospective study in 195 patients. Gut 33: 155-158, 1992.
10. Streitz JM, Ellis FH, Gibb SP and Heatley GM: Achalasia and squamous cell carcinoma of the esophagus: Analysis of 241 patients. Ann Thorac Surg 59: 1604-1609, 1995.

11. Tustumi F, Bernardo WM, da Rocha JRM, Szachnowicz S, Seguro FC, Bianchi ET, Sallum RAA and Cecconello I: Esophageal achalasia: A risk factor for carcinoma. A systematic review and meta-analysis. Dis Esophagus 30: 1-8, 2017.

12. Ghasemi-Kebria F, Joshaghani H, Taheri NS, Semnani S, Aarabi M, Salamat F and Roshandel G: Aflatoxin contamination of wheat flour and the risk of esophageal cancer in a high risk area in Iran. Cancer Epidemiol 37: 290-293, 2013.

13. Marchese S, Polo A, Ariano A, Velotto S, Costantini S and Severino L: Aflatoxin B1 and M1: Biological properties and their involvement in cancer development. Toxins (Basel) 24: E214, 2018.

14. Rawal S, Kim JE and Coulombe R Jr: Aflatoxin B1 in poultry: Toxicology, metabolism and prevention. Res Vet Sci 89: 325-331, 2010.

15. Raisuddin S, Singh K, Zaidi S, Paul BN and Ray PK: Immunosuppressive effects of aflatoxin in growing rats. Mycopathologia 124: 189-194, 1993.

16. Marin D, Taranu I, Bunaciu RP, Pascale F, Tudor DS, Avram N, Sarca M, Cureu I, Criste RD, Suta V and Oswald IP: Changes in performance, blood parameters, humoral and cellular immune responses in weanling piglets exposed to low doses of aflatoxin. J Anim Sci 80: 1250-1257, 2002.

17. Park JW, Kim EK, Shon DH and Kim YB: Natural co-occurrence of aflatoxin B1, fumonsin B1, and ochratoxin A in barley and maize foods from Korea. Food Addit Contam 19: 1073-1080, 2002.

18. Inoue H, Minami H, Kobayashi Y, Sato Y, Kaga M, Suzuki M, Satodate H, Odaka N, Itoh $\mathrm{H}$ and Kudo S: Peroral endoscopic myotomy (POEM) for esophageal achalasia. Endoscopy 42: 265-271, 2010.

19. Zhou PH, Li QL, Yao LQ, Xu MD, Chen WF, Cai MY, Hu JW, Li L, Zhang YQ, Zhong YS, et al: Peroral endoscopic remyotomy for failed heller myotomy: A prospective single-center study. Endoscopy 45: 161-166, 2013.

20. Khashab MA, Messallam AA, Onimaru M, Teitelbaum EN, Ujiki MB, Gitelis ME, Modayil RJ, Hungness ES, Stavropoulos SN, El Zein MH, et al: International multi-center experience with peroral endoscopic myotomy for the treatment of spastic esophageal disorders refractory to medical therapy (with video). Gastrointest Endosc 81: 1170-1177, 2015.

21. Jones EL, Meara MP, Pittman MR, Hazey JW and Perry KA: Prior treatment does not influence the performance or early outcome of per-oral endoscopic myotomy for achalasia. Surg Endosc 30: 1282-1286, 2016.

22. Inoue H, Sato H, Ikeda H, Onimaru M, Sato C, Minami H, Yokomichi H, Kobayashi Y, Grimes KL and Kudo SE: Per-Oral endoscopic myotomy: A series of 500 patients. J Am Coll Surg 221: 256-264, 2015.

23. Minami H, Yamaguchi N, Matsushima K, Akazawa Y, Ohnita K, Takeshima F, Nakayama T, Hayashi T, Inoue H, Nakao K and Isomoto $\mathrm{H}$ : Improvement of endocytoscopic findings after per oral endoscopic myotomy (POEM) in esophageal achalasia; does POEM reduce the risk of developing esophageal carcinoma? Per oral endoscopic myotomy, endocytoscopy and carcinogenesis. BMC Gastroenterol 30: 22, 2013.

24. Wang J and Liu XM: Assessment of dietary aflatoxin exposure in Chinese residents. Chin J Food Hyg 19: 238-240, 2007 (In Chinese).

25. Eckardt V: Clinical presentations and complications of achalasia. Gastrointest Endosc Clin N Am 11: 281-292, vi, 2001.

26. Pandolfino JE, Kwiatek MA, Nealis T, Bulsiewicz W, Post J and Kahrilas PJ: Achalasia: A new clinically relevant classification by high-resolution manometry. Gastroenterology 135: 1526-1533, 2008 .

27. Eckardt AJ and Eckardt VF: Treatment and surveillance strategies in achalasia: An update. Nat Rev Gastroenterol Hepatol 8: 311-319, 2011.

28. Ferri F, Brera C, De Santis B, Fedrizzi G, Bacci T, Bedogni L, Capanni S, Collini G, Crespi E, Debegnach F, et al: Survey on urinary levels of aflatoxins in professionally exposed workers. Toxins (Basel) 9: E117, 2017.

29. Yard EE, Daniel JH,Lewis LS, Rybak ME, Paliakov EM,Kim AA, Montgomery JM, Bunnell R, Abudo MU, Akhwale W, et al: Human aflatoxin exposure in Kenya, 2007: A cross-sectional study. Food Addit Contam Part A Chem Anal Control Expo Risk Assess 30: 1322-1331, 2013. 
30. Fujii T, Yamana H, Sueyoshi S, Fujita H, Tanaka Y, Kubota M Toh U, Mine T, Sasahara H, Shirouzu K, et al: Histopathological analysis of non-malignant and malignant epithelium in achalasia of the esophagus. Dis Esophagus 13: 110-116, 2000.

31. Leeuwenburgh I, Gerrits MM, Capello A, van den Bogert B, van Dekken H, Steyerberg EW, Siersema PD and Kuipers EJ: Expression of p53 as predictor for the development of esophageal cancer in achalasia patients. Dis Esophagus 23: 506-511, 2010

32. Peraica M, Radic B, Lucic A and Pavlović M: Toxic effects of mycotoxins in humans. Bull World Health Organ 77: 754-766, 1999.

33. Luzi A, Cometa MF and Palmery M: Acute effects of aflatoxins on guinea pig isolated ileum. Toxicol In Vitro 6: 525-529, 2002.

34. Gursoy N, Durmus N, Bagcivan I, Sarac B, Parlak A, Yildirim S and Kaya T: Investigation of acute effects of aflatoxin on rat proximal and distal colon spontaneous contractions. Food Chem Toxicol 46: 2876-2880, 2008.

35. Gockel HR, Schumacher J, Gockel I, Lang H, Haaf T and Nöthen MM: Achalasia: Will genetic studies provide insights? Hum Genet 128: 353-364, 2010.

36. Boeckxstaens GE: Achalasia: Virus-induced euthanasia of neurons? Am J Gastroenterol 103: 1610-1612, 2008.

37. Rohof WO, Salvador R, Annese V, Bruley des Varannes S, Chaussade S, Costantini M, Elizalde JI, Gaudric M, Smout AJ, Tack J, et al: Outcomes of treatment for achalasia depend on manometric subtype. Gastroenterology 144: 718-725, 2013.

38. Eckardt VF, Kohne U, Junginger T and Westermeier T: Risk factors for diagnostic delay in achalasia. Dig Dis Sci 42: 580-585, 1997.

39. El-Takli I, O'Brien P and Paterson WG: Clinical diagnosis of achalasia: How reliable is the barium x-ray? Can J Gastroenterol 20: $335-337,2006$
40. Ghoshal UC, Daschakraborty SB and Singh R: Pathogenesis of achalasia cardia. World J Gastroenterol 28: 3050-3057, 2012.

41. Moon EY and Pyo S: Aflatoxin B(1) inhibits CD14-mediated nitric oxide production in murine peritoneal macrophages. Int $\mathrm{J}$ Immunopharmacol 22: 237-246, 2000.

42. Moon EY, Rhee DK and Pyo S: Inhibition of various functions in murine peritoneal macrophages by aflatoxin $\mathrm{B} 1$ exposure in vivo. Int J Immunopharmacol 21: 47-58, 1999.

43. Moon EY, Han JJ, Rhee DK and Pyo S: Aflatoxin B1-induced suppression of nitric oxide production in murine peritoneal macrophages. J Toxicol Environ Health A 55: 517-530, 1998.

44. Khajanchee YS, VanAndel R, Jobe BA, Barra MJ, Hansen PD and Swanstrom LL: Electrical stimulation of the vagus nerve restores motility in an animal model of achalasia. J Gastrointest Surg 7: 843-849, 2003.

45. de Souza EM, Rivera MT, Araujo-Jorge TC and de Castro SL: Modulation induced by estradiol in the acute phase of Trypanosoma cruzi infection in mice. Parasitol Res 87: 513-520, 2001.

46. Ming $\mathrm{Z}$ and Davis $\mathrm{CD}: \mathrm{CD}^{+} \mathrm{T}$ lymphocytes required for enhanced survival of Trypanosoma cruzi-infected mice at elevated environmental temperature. J Parasitol 89: 630-632, 2003.

This work is licensed under a Creative Commons Attribution-NonCommercial-NoDerivatives 4.0 International (CC BY-NC-ND 4.0) License. 